\title{
Predicting Ecosystem Response to Perturbation from Thermodynamic Criteria
}

\author{
Vasthi Alonso Chávez ${ }^{1}$, Karo Michaelian² \\ ${ }^{1}$ School of Mathematics, University of Southampton, Southampton, UK \\ ${ }^{2}$ Institúto de Física, UNAM, México, Cto. de la Investigación Científica, Cuidad Universitaria, Mexico City, Mexico \\ E-mail: vastichu@gmail.com, karo@fisica.unam.mx \\ Received February 1, 2011; revised April 17, 2011; accepted May 5, 2011
}

\begin{abstract}
The response of ecosystems to perturbations is considered from a thermodynamic perspective by acknowledging that, as for all macroscopic systems and processes, the dynamics and stability of ecosystems is subject to definite thermodynamic law. For open ecosystems, exchanging energy, work, and mass with the environment, the thermodynamic criteria come from non-equilibrium or irreversible thermodynamics. For ecosystems during periods in which the boundary conditions may be considered as being constant, it is shown that criteria from irreversible thermodynamic theory are sufficient to permit a quantitative prediction of ecosystem response to perturbation. This framework is shown to provide a new perspective on the population dynamics of real ecosystems.
\end{abstract}

Keywords: Population Dynamics, Ecosystem Perturbation, Non-Equilibrium Thermodynamics, Enrichment Paradox

\section{Introduction}

Most ecosystems are under considerable stress, having been perturbed by human intrusions including, population reduction, the introduction of foreign species, habitat fragmentation, contamination, and general global warming. Fortunately, ecosystems can often recover from perturbations and can even evolve and adapt to new boundary conditions [1]. However, successful recovery depends on the inherent stability of the system, which is a complex function of the individual interactions among all the participating species and among species and their environment. Given that typical ecosystems contain over 3000 species [2], understanding the nature of this stability, and thus predicting ecosystem response to perturbation, is far from trivial, but indispensable for developing a quantitative approach to conservation.

Predicting ecosystem response to perturbation is, therefore, one of the most scientifically taxing yet important questions of our time. Most of present ecosystem dynamics theory is based on Lotka-Volterra-type equations which are empirically inspired equations incorporating one-body parameters, such as inherent birth and death rates, and two-body effects of one species population on another through a "community matrix" of coeffi- cients representing; competition, predator-prey, symbiosis, or neutral interaction. Environment limitations are incorporated through additional parameters such as the "carrying capacity". This two-body "community matrix" approach, although widely recognized for its usefulness in revealing the general spectrum of the dynamics of model ecosystems [3], has had limited application to predicting real ecosystem response to perturbation.

This is primarily due to the fact that the community matrix is obtained from fits to time-series population data, and therefore it can be expected to be representative of nature only within the limited range of the available population data.

A further problem debilitating the community matrix approach is that it is a two-body approach, while species interactions are really of a many-body nature. These many-body effects are usually absorbed within so called "environmental factors" which are included in the dynamical equations as fitted constants. However, these "constants" are not really constant for perturbed ecosystems and anyhow fail to endow the resultant 2-body equations with the true dynamics of inherently many-body natural ecosystems.

A lack of a quantitative ecosystem theory means that today's ecosystem health is usually surmised by making 
painstaking field counts of the populations of particular species and then, somehow arbitrarily, deciding whether or not to include those species on an "in danger of extinction" list. Such one dimensional vigilance of ecosystems is not satisfactory for a number of reasons: first, it fails to treat the ecosystem as an integrated whole and could thereby miscalculate the gravity of the situation about to unfold; second, since many ecosystems have a natural cyclical, or even chaotic, but stable dynamics, it may be difficult to distinguish normal stable periodic or chaotic behavior from a dangerous fall toward extinction; third, our human perspective tends to focus on species in which the individuals are physically large, easily observable, or likable, but not necessarily those key species that are most important to the stability of an ecosystem. Most important, however, is the fact that present ecosystem theory provides little information for designing an integral approach to arresting an impending catastrophe, other than, perhaps, suggesting that the endangered specie be protected by law.

There is clearly a need for attempts at constructing more quantitative approaches to population modeling based on fundamental science and measurements that can lead to prediction of ecosystem dynamics in regions in population space for which no data exist. There has been a growing realization that such a quantitative theory of ecosystems will ultimately have a thermodynamic basis [4-10]. The reasons are compelling: First, thermodynamic laws derive from symmetry principles inherent in nature and thus are universal, applicable in suitable form to all macroscopic systems and processes, irrespective of the types of interactions involved. Second, thermodynamics deals with a much reduced set of macroscopic variables which can be related with measurable ecosystem variables (e.g. populations) involved in the dynamical patterns observed in Nature. Third, the strictly hierarchal control assumed in traditional ecology is supplanted by a more integral approach in which systems are thermodynamically embedded [8,9,11]. Finally, a number of problems and paradoxes existing in traditional ecosystem theory appear to have a simple resolution in terms of thermodynamic directives $[7,10]$.

The objective of this paper is to demonstrate that, for particular ecosystems under constant boundary conditions, a non-equilibrium thermodynamic framework for the population dynamics can lead to explicit predictions concerning ecosystem response to perturbation. In the following section we briefly outline the thermodynamic framework for treating ecosystems which has been presented elsewhere [10]. In Section 3 we present a simple model ecosystem and demonstrate how its population dynamics and stability characteristics are determined by thermodynamic constraints and criteria relating to energy, work, and mass flow among the species populations, and with the external environment. In Section 4, we perturb this ecosystem and analyze the response as predicated on the basis of non-equilibrium thermodynamic formalism. Finally, in Section 5, we discuss how our thermodynamic framework may have relevance in explaining the particular population dynamics observed in many real ecosystems.

\section{Thermodynamic Framework}

To avoid misinterpretation at the outset, it is prudent to make a clear distinction between two existing, but fundamentally different, thermodynamic frameworks. Equilibrium thermodynamics deals with isolated systems and the fundamental state variable governing the evolution of the isolated system toward the stable equilibrium state is the total entropy, S. Irreversible thermodynamics deals with open systems or processes, such as ecosystems, which exchange energy, work, and mass between component parts and with the environment. Here, the variable governing the evolution toward the stable stationary state (for constant boundary conditions) is the time variation of the total entropy of the system, $\mathrm{d} S / \mathrm{d} t$. Our framework is based on the latter, irreversible thermodynamics, and we employ only that part of this framework, known as classical, developed by Lars Onsager [12] and Illya Prigogine [13], which has been extensively verified empirically.

As for any open system, the time variation of the total entropy of the ecosystem may be divided into a part due to the internal entropy production arising from irreversible processes occurring within the ecosystem itself, and a second part due to the flow of entropy into, or out of, the ecosystem from the external environment [13],

$$
\frac{\mathrm{d} S}{\mathrm{~d} t}=\frac{\mathrm{d}_{i} S}{\mathrm{~d} t}+\frac{\mathrm{d}_{e} S}{\mathrm{~d} t} .
$$

All macroscopic systems and processes, including ecosystems, are subject to definite thermodynamic law. The primary among these is the second law of thermodynamics which states that the internal production of entropy due to irreversible processes occurring within the system must be positive definite,

$$
\frac{\mathrm{d}_{i} S}{\mathrm{~d} t}>0
$$

For the case of ecosystems under the condition of constant external constraints (see [10] for justification of this condition for a large class of ecosystems), classical irreversible thermodynamic theory states [13] that the system will eventually arrive at a thermodynamic stationary state in which all macroscopic variables, including the total entropy, are stationary in time, 


$$
\frac{\mathrm{d} S}{\mathrm{~d} t}=0 .
$$

Therefore, from (2.1), at the stationary state,

$$
\frac{\mathrm{d}_{i} S}{\mathrm{~d} t}=-\frac{\mathrm{d}_{e} S}{\mathrm{~d} t},
$$

implying from Equation (2.2) that

$$
\frac{\mathrm{d}_{e} S}{\mathrm{~d} t}<0 \text {. }
$$

Maintaining an ecosystem in a stable thermodynamic stationary state thus requires a continuous negative flow of entropy into the system. This was emphasized by Schrödinger [14], but was first recognized by Boltzmann [15].

The internal entropy production $\mathrm{d}_{i} S / \mathrm{d} t$ can be written as a sum of generalized thermodynamic forces $X$ multiplied by their corresponding generalized thermodynamic flows $J$, as stated by Prigogine [13]

$$
\frac{\mathrm{d}_{i} S}{\mathrm{~d} t}=\sum_{\alpha} X_{\alpha} J_{\alpha},
$$

(for example, the entropy production due to heat flow can be obtained by multiplying $X_{Q}=\nabla(1 / T)$ gradient of the inverse temperature, and $J_{Q}=$ heat flow).

The separation of the entropy production into its components of thermodynamic forces and flows is somewhat arbitrary and can often be chosen for convenience in resolving a specific problem. However, there are a number of conditions that must be met for any particular choice. The first condition is that the product of the force and flow gives units of entropy production, and the second is that symmetry aspects must be respected, for example, a scalar force cannot give rise to a vector flow [16]. We have shown [10] that ecosystem dynamics can be treated consistently within this irreversible thermodynamic framework by assigning the generalized thermodynamic forces to the species populations $\left(X_{\alpha} \equiv p_{\alpha}\right.$ ) (where $\alpha$ represents the species type) and the generalized flows to the flows of entropy ( $J_{\alpha} \equiv \mathrm{d} S_{\alpha} / \mathrm{d} t$ ) (due to flows of energy, work, or mass, to or from species $\alpha$, see below).

Within this framework, it was shown [10] that the ecological steady state, prevalent in nature $[2,17]$, has the stability characteristics of the thermodynamic stationary state. In view of this, we have made the formal assertion that the ecological steady state is just a particular case of the more general thermodynamic stationary state [10].

A further criterion from classical irreversible thermodynamic theory, considered by Prigogine as the most general result of irreversible thermodynamic theory, and valid for constant external constraints, is that the rate of change of the internal entropy production, due to changes in the generalized forces $X$ (the populations), is negative semi-definite; the general evolutionary criterium [13],

$$
\frac{\mathrm{d}_{X} \wp}{\mathrm{d} t} \leq 0 \quad \text { where } \wp \equiv \frac{\mathrm{d}_{i} S}{\mathrm{~d} t} .
$$

Equation (2.7) implies that, under constant boundary conditions, all natural changes in the species populations must be in such a manner so as to reduce the internal production of entropy. This is a powerful auxiliary criterion on ecosystem response to perturbation and it will be shown in $\S 3$ and that this, together with the second law of thermodynamics, and the fact that a system with constant external constraints must arrive at a thermodynamic stationary state, effectively determines the population dynamics that the ecosystem can assume. In this manner, we can predict the dynamical response of the ecosystem to perturbation, be it either toward recovery, toward a new dynamics, or toward extinction.

\section{Model Ecosystem}

We now present our thermodynamic framework for an over simplified but illustrative 3-species model ecosystem, including up to 3-body interaction terms. Two of the populations, $p_{1}$ and $p_{2}$, are considered variable, while the third, $p_{3}$ is fixed, and represents the constant boundary conditions over the ecosystem; such as the constant supply of nutrients due to a primary producer species.

The total entropy brought into the ecosystem or carried out of it through one-body transport processes can be written as,

$$
\frac{\mathrm{d}_{e} S}{\mathrm{~d} t}=\sum_{\alpha=1}^{n} p_{\alpha} \Gamma_{\alpha}^{e}
$$

where the sum is over all $n=3$ species and $p_{\alpha}$ is the population of species $\alpha . \Gamma_{\alpha}^{e}$ represents the average rate of exchange, or flow, of entropy with the external environment per individual per unit time of species $\alpha$ as a result of energy (including heat), work, or matter flow (see below).

Similarly, the internal entropy production, including production and exchange of entropy between individuals of the species, may be written as a many-body expansion [10],

$$
\begin{aligned}
\wp & \equiv \frac{d_{i} S}{d t} \\
& =\sum_{\alpha=1}^{n} p_{\alpha}\left[\Gamma_{\alpha}+\sum_{\alpha^{\prime}=1}^{n} p_{\alpha^{\prime}} \Gamma_{\alpha \alpha^{\prime}}+\sum_{\alpha^{\prime}, \alpha^{\prime \prime}=1}^{n} p_{\alpha^{\prime}} p_{\alpha^{\prime \prime}} \Gamma_{\alpha \alpha^{\prime} \alpha^{\prime \prime}}+O(4)\right] \\
& >0 .
\end{aligned}
$$

The $\Gamma_{\alpha}$ represent the entropy production per indi- 
vidual of species $\alpha$ due to one-body irreversible processes occurring within the individual such as; photosynthesis, transpiration, respiration, metabolism, etc. The $\Gamma_{\alpha \alpha^{\prime}}$ represent the entropy production and exchange due to 2-body interactions between individuals of species $\alpha$ and $\alpha^{\prime}$ (e.g. those involved in competition, predator-prey, symbiosis, etc.); $\Gamma_{\alpha \alpha^{\prime} \alpha^{\prime \prime}}$ corresponds to similar but 3-body interactions, and $O(4)$ represents the entropy production due to 4-body and higher order interactions (for example, those required for the functioning of societies). The 4-body and higher order N-body terms will be neglected in what follows since they would normally be small as they require increasingly improbable (except for social species) N-body localization in space and time.

Equation (2.7), for the time change in the entropy production due to a change in the generalized forces $X$ (the populations) then becomes,

$$
\begin{aligned}
\mathrm{d}_{\chi} \wp & =\sum_{\alpha} \mathrm{d} p_{\alpha}\left[\Gamma_{\alpha}+\sum_{\alpha^{\prime}} p_{\alpha^{\prime}} \Gamma_{\alpha \alpha^{\prime}}+\sum_{\alpha^{\prime} \alpha^{\prime \prime}} p_{\alpha^{\prime}} p_{\alpha^{\prime \prime}} \Gamma_{\alpha \alpha^{\prime} \alpha^{\prime \prime}}\right] \\
& \leq 0 .
\end{aligned}
$$

The dynamics of the ecosystem can now be determined from Equations (2.4), (3.1), (3.2) and (3.3) once the $\Gamma^{\prime} s$ are specified.

The $\Gamma$ 's represent entropy production and flow between individuals of the species and between individuals and their environment. A general expression for this entropy flow comes from the Gibb's equation and results from the flow of energy, work, and matter [13]. For example, the energy per individual per unit time taken in through photosynthesis $\mathrm{d} e_{\alpha}$, or the heat $\mathrm{d} q_{\alpha}$ per individual per unit time transported to the external environment, the work done on the environment per unit time $P \mathrm{~d} V_{\alpha}$ at constant pressure $P$, and the matter components (e.g. nutrients) of type $\beta$ taken in or given out by species $\alpha, d n_{\alpha \beta}$, of chemical potential $\mu_{\alpha \beta}$, give for the rate of entropy exchange per individual with the environment,

$$
\begin{aligned}
\Gamma_{\alpha}{ }^{e}= & \frac{1}{T} \frac{\mathrm{d} e_{\alpha}+\mathrm{d} q_{\alpha}}{\mathrm{d} t}+\frac{P}{T} \frac{\mathrm{d} V_{\alpha}}{\mathrm{d} t} \\
& -\frac{1}{T} \sum_{\beta} \mu_{\alpha \beta} \frac{\mathrm{d} n_{\alpha \beta}}{\mathrm{d} t}
\end{aligned}
$$

where the temperature $T$ (of the participating individuals) may be approximated as being constant for the ecosystem [5]. A similar expression can be written for the $\Gamma_{\alpha \alpha^{\prime}}$, representing the entropy production and exchange between individuals of species $\alpha$ and $\alpha^{\prime}$, i.e. in terms of the energy, work, and matter exchanged due to the 2-body interactions between individuals of the species. The effect on the entropy flows due to a simultaneous interaction of a third individual (three-body effects) of species $\alpha^{\prime \prime}$ is considered in the parameter $\Gamma_{\alpha \alpha^{\prime} \alpha^{\prime \prime}}$.

Determining the $\Gamma^{\prime} s$ for a real ecosystem therefore requires the determination of the flows of energy de, heat $\mathrm{d} q$, volume $\mathrm{d} V$, and mass $\mathrm{d} n_{\beta}$ of type $\beta$ between individuals of the participating species and between individuals and the external environment. Such a determination is possible in principle but obviously difficult in practice. Some of the experimental details for obtaining these types of flow measurements between the individuals, and between the individuals and their environment, can be found in [5] and references therein, as well as in $[18,19]$.

The entropy production due to the interaction between individuals $\Gamma_{\alpha \alpha^{\prime}}$ is difficult to measure. However, much work has already been performed on determining the energy flow in ecosystems [20,21] and, as Equation (3.4) shows, determining the entropy flow requires merely extending this program to determining also mass [21] and work flow between individuals, and between individuals and the environment.

In the absence of real ecosystem data concerning the production of entropy and the exchange of entropy, here we generate these coefficients $\Gamma$ for a model ecosystem subject to thermodynamic law with the aid of a genetic algorithm [22]. The algorithm begins by randomly generating sets of initial values for the entropy production and exchanges, the $\Gamma^{\prime} s$, within fixed ranges. The algorithm then evaluates the fitness of each set by checking to see if the population dynamics, as determined by criteria (3.2) and (3.3), leads to a viable stationary state in the long time limit, i.e. one with $\lim \mathrm{d}_{i} S / \mathrm{d} t=-\mathrm{d}_{e} S / \mathrm{d} t$ (as required by classical irre-

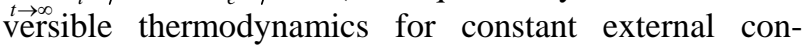
straints, see Equation (2.4)) and with $\mathrm{d}_{i} S / \mathrm{d} t$ large and positive (consistent with what is known about the natural evolution of biotic systems to ever higher entropy production regimes (see $[6,13])$. The best sets of $\Gamma^{\prime} s$ are selected and evolved through mutation and crossover, optimizing (maximizing), the fitness function,

$$
\frac{\mathrm{d}_{i} S / \mathrm{d} t}{\mathrm{~d}_{i} S / \mathrm{d} t+\mathrm{d}_{e} S / \mathrm{d} t},
$$

which, as required, is large for $\mathrm{d}_{i} S / \mathrm{d} t$ large, and for $\lim _{t \rightarrow \infty} \mathrm{d}_{i} S / \mathrm{d} t=-\mathrm{d}_{e} S / \mathrm{d} t$.

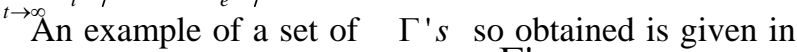
Appendix A. Using this set of $\Gamma^{\prime} S$ and a starting population set of populations $\left(p_{1}=1000, p_{2}=2000\right)$ with $p_{3}$ fixed at 2000 (the constant external constraint), and generating infinitesimal variations of the populations $\mathrm{d} p_{1}$ and $\mathrm{d} p_{2}$ at random $\left(\mathrm{d} p_{3}=0\right)$ while only accepting those sets $\{d p\}$ which satisfy the thermodynamic criteria of Equations (3.2) and (3.3), leads to the stable cyclic attractor (oscillating) population dynamics as 
shown in Figure 1.

\section{Response to Perturbation}

The response to perturbation obtained under the dictates of the thermodynamic criteria, Equations (3.2) and (3.3), once the ecosystem has arrived at a stationary state, Equation (2.4), are shown in Figure 1. This stationary state, for the particular set of $\Gamma$ 's obtained, is a cyclic attractor. The first perturbation, affected at time $k=5 e 6$ by reducing the population of $p_{1}$ to 40 , can be seen to have little effect on the ecosystem, a full recovery of the populations is obtained rapidly. The second perturbation affected by increasing the population of $p_{1}$ to 800 at time $k=8 e 6$ also produces only a small transient effect. However, if the ecosystem is perturbed in the same manner but at time $k=1.3 e 7$, population $p_{1}$ goes negative (as does the internal production of entropy $\mathrm{d}_{i} S / \mathrm{d} t$ ) implying the extinction of the species and the thermodynamic non-viability of the ecosystem. The timing of a perturbation affected on an ecosystem with cyclic attractor population dynamics therefore appears to be crucial in deciding the fate of the ecosystem.

Similar results are obtained if population $p_{2}$ is perturbed. A perturbation of the ecosystem at time $k=1.7 e 7$ by increasing population $p_{2}$ to 1600 has no long term consequences. However, when population $p_{2}$ is reduced to 600 at time $k=2.0 e 7$, the internal production of en- tropy and the population $p_{1}$ both go negative, the ecosystem again becomes untenable.

Figure 2 plots the dynamics of the ecosystem in population space $p_{1}: p_{2}$ for 50 different initial populations. It is apparent from this figure that the recovery or not of an ecosystem from a particular perturbation depends on the region in population space into which the ecosystem is perturbed.

Perturbation into the "regions of danger" marked on the figure leads to either one of the populations going negative, or to the internal production of entropy going negative. Both of these results are non physical and would foretell the collapse of the ecosystem in nature. Interestingly, these regions of danger do not necessarily correspond to regions of small population.

We next examine the response of our model ecosystem to permanent changes in the boundary conditions. Figure 3 shows the ecosystem dynamics under new boundary conditions of $p_{3}$ reduced to 200, down from its originally fixed value of $p_{3}=2000$, implying a less negative flow of entropy into the ecosystem. Without allowing time for the interaction coefficients $\Gamma$ to evolve in response to the new boundary conditions, the cyclic attractor then becomes a point attractor as shown in Figure 3. However, as can be seen from this figure, the point attractor is not a thermodynamic stationary state since the internal production of entropy is no longer equal to the negative of the external flow of entropy (Equation
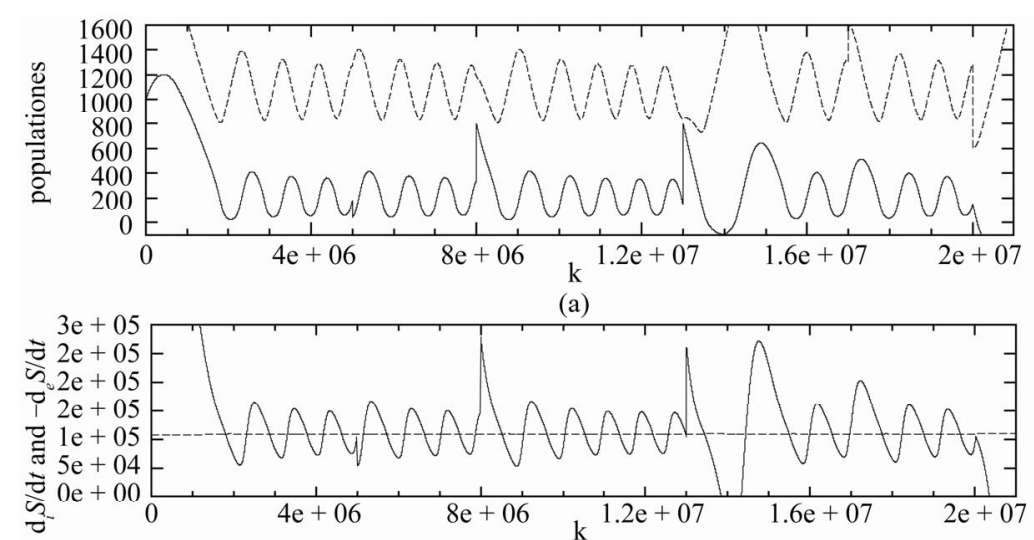

(b)

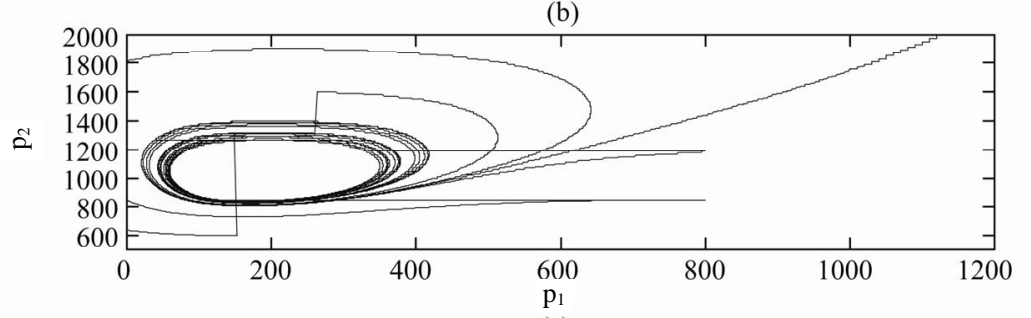

(c)

Figure 1. (a) Populations $p_{1}$ (solid line) and $p_{2}$ (dashed line) as a function of time $k$ for the ecosystem given in Appendix $A$ in response to various (see text) perturbations. (b) $\mathrm{d}_{i} S / \mathrm{d} t$ (solid line) and $-\mathrm{d}_{e} S / \mathrm{d} t$ (dashed line) as a function of time. (c) Trajectory in population space showing the cyclic attractor dynamics and the effects of 4 distinct perturbations (see text). 


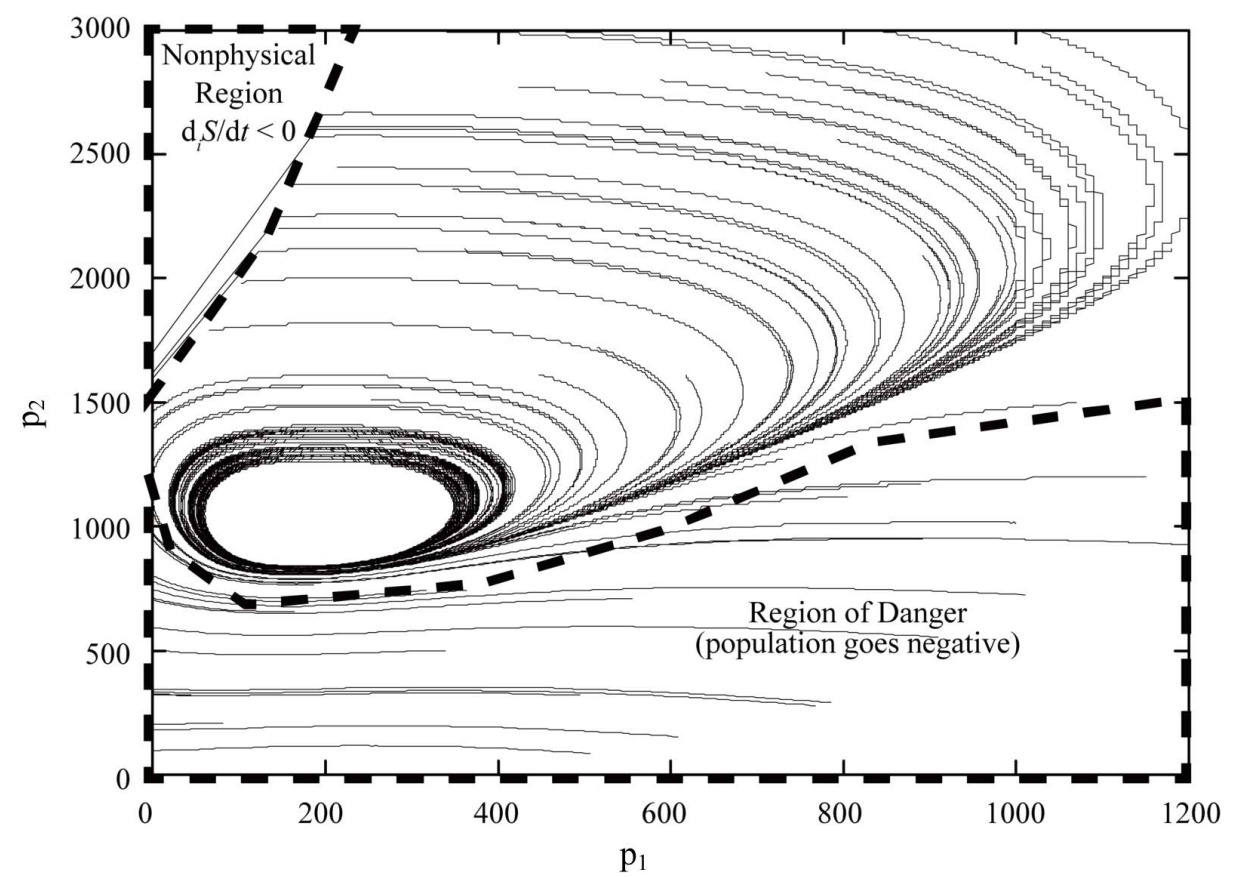

Figure 2. The dynamics in population space for 50 different initial populations showing that there are "regions of danger" in population space for which a perturbation into these regions would cause the system to become untenable, either because one of the populations extinguishes (goes negative) or because the internal production of entropy becomes negative (nonphysical).
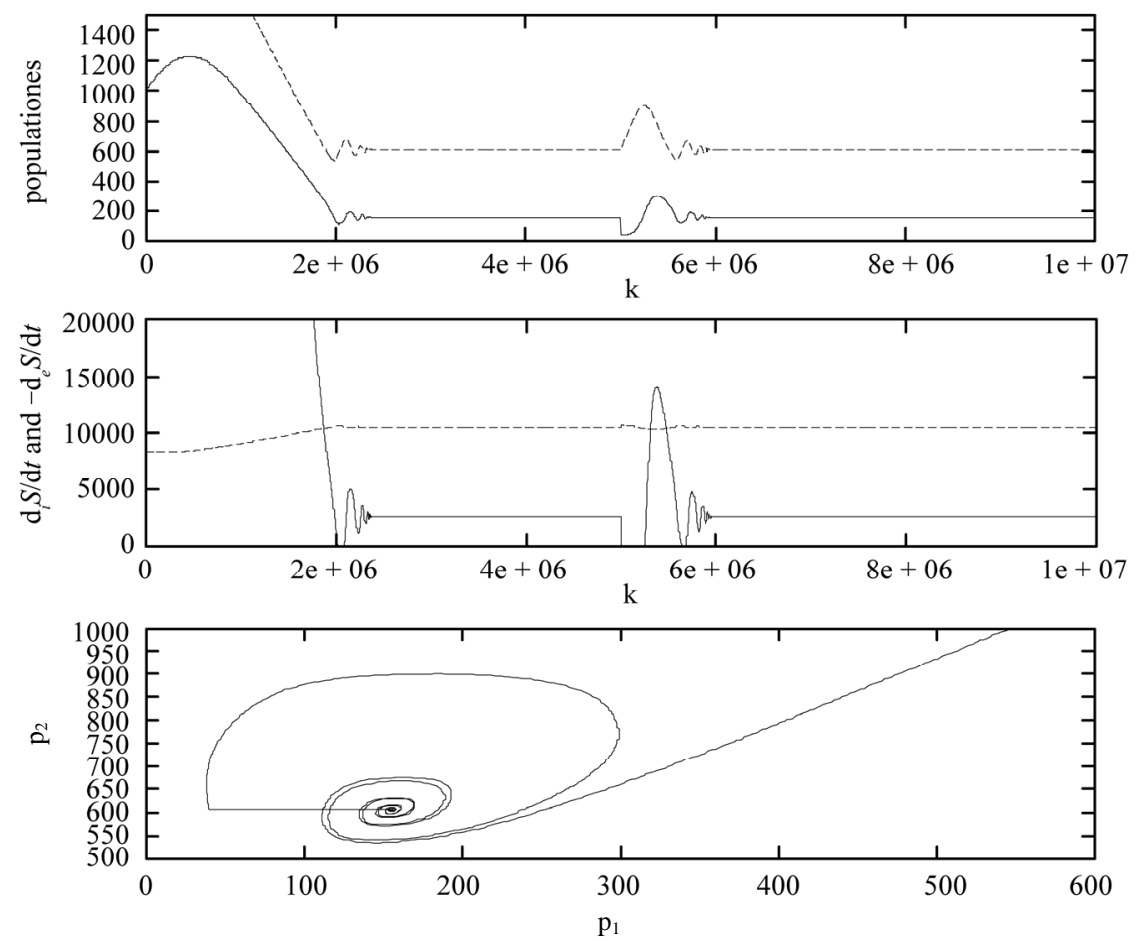

Figure 3. Ecosystem dynamics for the case where the fixed boundary conditions have been changed from $p_{3}=2000$ to $p_{3}=200$. The population dynamics becomes that of a point attractor. However, the system is not in a thermodynamic stationary state since $\mathrm{d}_{i} S / \mathrm{d} t \neq-\mathrm{d}_{e} S / \mathrm{d} t$. A subsequent perturbation of $p_{1}$ to 40 at $k=5 e 6$, which did not affect the stability previously (see Figure 1), now leads to $d_{i} S / d t$ going negative, violating the 2 nd law of thermodynamics. The vulnerability of the ecosystem has thus been increased by reducing the external constraint of $p_{3}$. 
(2.4) is no longer satisfied). The ecosystem fitness function, Equation (3.5), is no longer at a local maximum value and the system, given time, would evolve its entropy production and exchange coefficients (the set $\Gamma$ ) until reaching a new stationary state where the production and external flow of entropy are once again equal. Note that in the perturbed state with the new external constraint, $p_{3}=200$, the same small perturbation of reducing the population $p_{1}$ to 40 at time $k=5 e 6$, which did not have any lasting affect on the ecosystem previously (Figure 1), now results in the collapse of the ecosystem since it moves it into the non-physical, thermodynamiccally prohibited, regime of negative internal entropy production (Figure 3).

Figure 4 shows the opposite effect of increasing the flow of negative entropy into the ecosystem, obtained by increasing the value of the fixed external condition to $p_{3}$ $=2200$. Without allowing time for the interaction coefficients $\Gamma$ to evolve, the dynamics remains that of a cyclic attractor but the orbit of the attractor increases significantly, bringing the population $p_{1}$ very close to zero at one point in its orbit. A slight perturbation of population $p_{2}$ at time $k=5 e 6$ is sufficient to cause the population $p_{1}$ to pass through zero and thereby cause the collapse of the ecosystem. We believe that this is a possible thermodynamic explanation of the "enrichment paradox"[23]; contrary to naïve expectation, an increase in the inflow of nutrients is often observed to make an eco- system more vulnerable to perturbation. This will be considered in detail in a forthcoming paper [24].

We have also verified [25] that had the set of $\Gamma$ 's been chosen such that the thermodynamic stationary state corresponded to a point attractor in population space (sometimes referred to as an equilibrium state in the ecological literature), then increasing the value of the fixed external condition $p_{3}$ leads to population oscilla- tions of $p_{1}$ and $p_{2}$. This may have relevance to the sudden outbreak of large population oscillations of certain spe- cies.

\section{Summary and Conclusions}

Acknowledging that ecosystems, like all macroscopic processes, are subject to definite thermodynamic law, we have demonstrated that under constant external constraints, the response of ecosystems to perturbation can, in principle, be predicted. The thermodynamic criteria which direct the dynamics come from non-equilibrium thermodynamic theory. They are; 1 ) the system must eventually arrive at a thermodynamic stationary state, Equation (2.4), 2) the internal production of entropy must be positive definite, in accord with the second law of thermodynamics, Equation (2.2), and 3) any natural change in the populations must be in such a manner so as to reduce the internal production of entropy of the entire system, Prigogine's general evolution criterion, Equation (2.7).
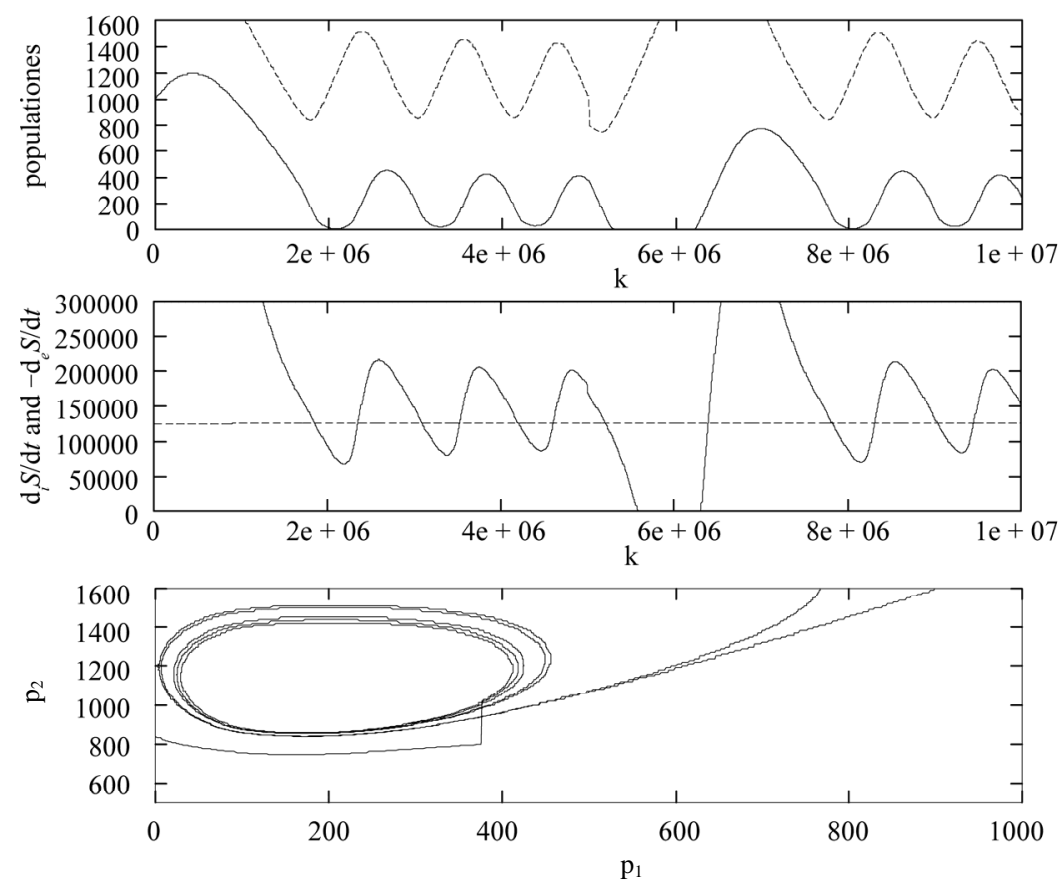

Figure 4. Ecosystem dynamics for the case where the fixed boundary condition has been increased from $p_{3}=2000$ to $p_{3}=2200$. In this case, the population dynamics is that of a cyclic attractor with a larger orbit, bringing $p_{1}$ very close to zero at times. $A$ subsequent small perturbation of $p_{2}$ to 800 at $k=5 e 6$ leads to $d_{i} S / d t$ going negative and population $p_{1}$ passing through zero. We believe that this is the thermodynamic origin of the "enrichment paradox" [23]. 
In the absence of data on the entropy production and exchange of real ecosystems, we considered a simple model ecosystem generated by evolving interaction coefficients (representing entropy production and exchange) through selection with a fitness function favoring the thermodynamic criteria identified above. We then studied the response of this model ecosystem to perturbation of the populations under the same thermodynamic criteria. We found that there exist regions in population space for which perturbation into these regions leads to the eventual extinction of one or more of the species, or to a negative internal production of entropy. The latter violates the second law of thermodynamics and would lead to ecosystem collapse since physical maintenance processes require positive production of entropy. An important finding is that these regions in population space are a general feature of the thermodynamic framework and do not necessarily correspond to regions of small population. Assigning species to "in danger of extinction" lists, solely on the basis of the smallness of their populations may therefore not be an effective conservation policy. Our proposed approach, based on thermodynamic criteria, predicts the dynamics over all of population space and thus leads to quantitative elements for providing more informed policies for responding to ecosystem perturbation.

Increasing or decreasing the negative flow of entropy (natural resources) into the ecosystem has the effect of increasing or decreasing respectively both the amplitude of the orbit of the attractor in population space and the internal production of entropy of the system. In either case, this results in a more vulnerable ecosystem since the populations pass closer to zero or the internal production of entropy may more easily become negative respectively. We believe that this result gives a thermodynamic explanation of the "enrichment paradox".

Ecosystems are composed of many thousands of interacting species and unless the ecosystem can be reduced to a few key species, the details of the dynamics is, undoubtedly, significantly more complicated than the results obtained here. However, our thermodynamic framework can be straightforwardly applied to a much larger and more complex ecosystem simply by measuring all the entropy production and exchange coefficients $\Gamma$ for all the species involved. Work in this direction is underway [24].

In conclusion, as for all macroscopic processes, ecosystems are subject to definite thermodynamic law. For constant external constraints, these laws are sufficient to determine ecosystem response to perturbation. Our analysis of the population dynamics based on thermodynamic law and the formulation of the interaction coeffi- cients in terms of physical and measurable quantities (the production and exchange of entropy) is one step toward a more quantitative theory of ecosystems.

\section{Acknowledgements}

K. M. is grateful for the financial support of DGAPAUNAM project numbers IN-118206, and IN- 112809, and for financial assistance while on a 2007 sabbatical leave. The hospitality afforded during this leave by the Faculty of Science, Universidad Autonoma del Estado de Morelos, Mexico, is greatly appreciated.

\section{References}

[1] J. Norberg, D. P. Swaney, J. Dushoff, J. Lin, R. Casagrandi and S. A. Levin, "Phenotypic Diversity and Ecosystem Functioning in Changing Environments: A Theoretical Framework," Proceedings of the National Academy of Sciences, Vol. 98, No. 20, 2001, pp. 11376-11381. doi:10.1073/pnas.171315998

[2] G. A. Polis, "Complex Trophic Interactions in Deserts: An Empirical Critique of Food Web Theory," American Naturalist, Vol. 138, No. 1, 1991, pp. 123-155. doi:10.1086/285208

[3] R. M. May, "Stability and Complexity in Model Ecosystems," 2nd Edition, Princeton University Press, Princeton, 1974.

[4] E. P. Odum, "The Strategy of Ecosystem Development," Science, Vol. 164, No. 3877, 1969, pp. 262-270. doi:10.1126/science.164.3877.262

[5] V. F. Gallucci, "On the Principles of Thermodynamics in Ecology," Annual Review of Ecology, Evolution, and Systematics, Vol. 4, 1973, pp. 329-357. doi:10.1146/annurev.es.04.110173.001553

[6] R. E. Ulanowicz and B. M. Hannon, "Life and the Production of Entropy," Proceedings of the Royal Society of London, Series B, Vol. 232, No. 1267, 1987, pp. 181-192. doi:10.1098/rspb.1987.0067

[7] R. Swenson, "Emergent Attractors and the Law of Maximum Entropy Production: Foundations to a Theory of General Evolution,”Systems Research, Vol. 6, No. 3, 1989, pp. 187-198.doi:10.1002/sres.3850060302

[8] S. N. Nielsen and R. E. Ulanowicz, "On the Consistency between Thermodynamical and Network Approaches to Ecosystems,” Ecological Modelling, Vol. 132, No. 1-2, 2000, pp. 23-31.doi:10.1016/S0304-3800(00)00302-1

[9] S. N. Nielsen, "Thermodynamics of an Ecosystem Interpreted as a Hierarchy of Embedded Systems,” Ecological Modelling, Vol. 135, No. 2-3, 2000, pp. 279-289. doi:10.1016/S0304-3800(00)00379-3

[10] K. Michaelian, "Thermodynamic Stability of Ecosystems,” Journal of Theoretical Biology, Vol. 237, No. 3, 2005, pp. 323-335. doi:10.1016/j.jtbi.2005.04.019

[11] R. E. Ulanowicz, "Ecology, the Ascendent Perspective," Columbia University Press, Columbia, 1997.

[12] L. Onsager, "Reciprocal Relations in Irreversible Proc- 
esses. I,” Physical Review, Vol. 37, No. 4, 1931, pp. 405-426; "Reciprocal Relations in Irreversible Processes. II,” Physical Review, Vol. 38, No. 12, 1931, pp. 2265-2279. doi:10.1103/PhysRev.38.2265

[13] I. Prigogine, "Thermodynamics of Irreversible ProceSses,” Wiley, New York, 1967.

[14] E. Schrödinger, "What Is Life?” Cambridge University Press, Cambridge, 1944.

[15] L. Boltzmann and B. Mcginnes, "Theoretical Physics and Philosophical Problems: Selected Writings," Kluwer Academic Publishers, Dordrecht, 1974.

[16] A. Katchalsky and P. F. Curran, "Nonequilibrium Thermodynamics in Biophysics," 4th Edition, Harvard University Press, Cambridge, 1975.

[17] L. Glodwasser and J. Roughgarden, "Construction and Analysis of a Large Caribbean Food Web,” Ecology, Vol. 74, No. 4, 1993, pp. 1216-1233. doi:10.2307/1940492

[18] N. Hernández, "Determination of Entropy Production in Plants,” Bachelor's Thesis, Universidad Nacional Autónoma de México, Mexico City, 2008.

[19] N. Hernández and K. Michaelian, "Determination of Entropy Production in Plants," 2010, Article under Preparation.
[20] M. Homer, W. M. Kemp and H. Mckellar, "Trophic Analysis of an Estuarine Ecosystem: Salt Marsh-Tidal Creek System Near Crystal River, Florida,” Manuscript, Department of Environmental Engineering, University of Florida, Gainesville, 1976.

[21] E. Halfon, N. Schito and R. E. Ulanowicz, "Energy Flow Through the Lake Ontario Food Web: Conceptual Model and an Attempt at Mass Balance,” Ecological Modelling, Vol. 86, No. 1, 1996, pp. 1-36. doi:10.1016/0304-3800(94)00195-2

[22] K. Michaelian, "Evolving Few-Ion Clusters of $\mathrm{Na}$ and Cl,” American Journal of Physics, Vol. 66, No. 3, 1998, pp. 231-240. doi:10.1119/1.18851

[23] M. Rosenzweig, "Paradox of Enrichment: Destabilization of Exploitation Ecosystems in Ecological Time,” Science, Vol. 171, No. 3969, 1971, pp. 385-387. doi:10.1126/science.171.3969.385

[24] V. Alonso Chávez and K. Michaelian, "Thermodynamic Origin of the Enrichment Paradox,” 2010, Article under Preparation.

[25] V. Alonso Chávez, "Respuesta de Ecosistemas a Perturbaciones dentro del Marco de la Termodinámica Irreversible," Masters of Science Thesis, Universidad Nacional Autónoma de México, Mexico City, 2007. 